\title{
Estimation of Multiple-Regime Threshold Autoregressive Models with Structural Breaks - Supplementary Materials
}

July 28, 2014

\section{S.1 Additional Simulations: TAR Model With One Structural Break}

In this example we study the following TAR model with one structural break

$$
x_{t}=\left\{\begin{array}{lc}
\left(-0.7 x_{t-1}-0.9 x_{t-2}+0.2 x_{t-3}\right) I_{(-\infty,-0.8)}+\left(0.8 x_{t-1}-0.3 x_{t-2}\right) I_{(-0.8,-0.3)} \\
-1.25 x_{t-1} I_{(-0.3, \infty)}+e_{t}, & 1 \leq t \leq 1250, \\
\left(0.5 x_{t-1}-0.3 x_{t-2}\right) I_{(-\infty,-1)}-0.6 x_{t-1} I_{(-1, \infty)}+e_{t}, & 1250<t \leq 2000 .
\end{array}\right.
$$

where $e_{t} \stackrel{i i d}{\sim} N(0,1)$ and $I_{(a, b)}=I\left(a<x_{t-1} \leq b\right)$. A realization of $(\mathrm{S} .1)$ is shown in Figure 1.

The proposed procedure in Section 3 is applied to 500 realizations of (S.1). Table 1 lists the percentages of the fitted number of segments. All the 500 realizations have the number of breaks correctly identified. Table 1 also reports the means and standard errors of the breakpoints.

Next, we checked the estimated numbers and locations of the thresholds. Table 2 summarizes the estimation results for the threshold parameters in the two segments. Note that the proposed procedure performs very well at locating both the structural breakpoints and thresholds. In fact, of the 500 realizations, $99.8 \%$ of them have the number of structural breaks and thresholds correctly identified.

\section{S.2 Proof of Theorem 2}

\section{Proof of Theorem 2.}




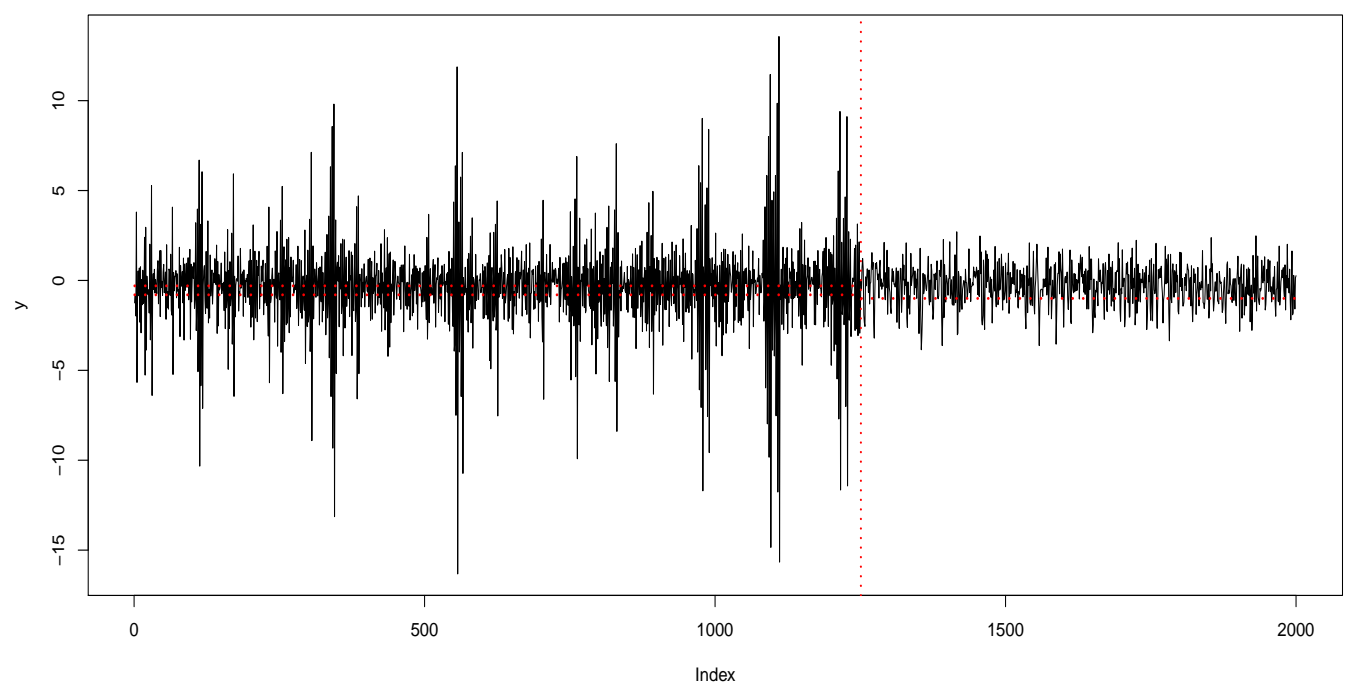

Figure 1: A realization generated from the process (S.1). The vertical dash line indicates the true breakpoint location, at $t=1250$.

Table 1: Estimated numbers and locations of breakpoints for the process (S.1).

\begin{tabular}{cccc}
\hline \hline number of & & \multicolumn{2}{c}{ break location } \\
segments & $\%$ & mean & s.e. \\
\hline 2 & 100.0 & 1251.50 & 6.198 \\
\hline
\end{tabular}

First we define some notations. For any $r, p, d \in \mathbb{Z}, \boldsymbol{\theta}=\left(\theta^{(0)}, \cdots, \theta^{(r+1)}\right)$, where $-\infty=\theta^{(0)}<$ $\theta^{(1)}<\cdots<\theta^{(r+1)}=\infty, \Psi=\left(\Psi_{1}, \ldots, \Psi_{r+1}\right), \Psi_{j}=\left(\sigma_{j}^{2}, \Phi_{j}\right)$ and $\boldsymbol{X}_{t-1}=\left(1, X_{t-1}, \ldots, X_{t-p}\right)$,

Table 2: Estimated number and values of thresholds for the process (S.1). The means of the estimated thresholds $\hat{\theta}_{i j}$ are reported when the number of thresholds were correctly identified. Standard errors are in parentheses.

\begin{tabular}{|c|c|c|c|c|c|}
\hline \multirow{2}{*}{$\begin{array}{l}\text { number of } \\
\text { thresholds }\end{array}$} & \multicolumn{3}{|c|}{ first segment } & \multicolumn{2}{|c|}{ second segment } \\
\hline & $\%$ & $\hat{\theta}_{11}$ & $\hat{\theta}_{12}$ & $\%$ & $\hat{\theta}_{21}$ \\
\hline 1 & 0 & & & 99.8 & $\begin{array}{c}-0.994 \\
(0.054)\end{array}$ \\
\hline 2 & 100 & $\begin{array}{l}-0.801 \\
(0.010)\end{array}$ & $\begin{array}{l}-0.294 \\
(0.050)\end{array}$ & 0.2 & \\
\hline 3 & 0 & & & 0 & \\
\hline$\geq 4$ & 0 & & & 0 & \\
\hline
\end{tabular}


define

$$
\begin{aligned}
& L_{n}\left(\lambda_{i-1}, \lambda_{i}, r, \boldsymbol{\theta}, \boldsymbol{\Psi}, p, d\right) \\
= & \sum_{t=\left[n \lambda_{i-1}\right]+1}^{\left[n \lambda_{i}\right]} \sum_{j=1}^{r+1}\left(\log \left(2 \pi \sigma_{j}^{2}\right)+\frac{\left(X_{t}-\Phi_{j}^{\prime} \boldsymbol{X}_{t-1}\right)^{2}}{\sigma_{j}^{2}} I\left(\theta^{(j-1)} \leq X_{t-d}<\theta^{(j)}\right)\right),
\end{aligned}
$$

and

$$
L\left(\lambda_{i-1}, \lambda_{i}, r, \boldsymbol{\theta}, \boldsymbol{\Psi}, p, d\right)=\lim _{n \rightarrow \infty} \frac{1}{n} L_{n}\left(\lambda_{i-1}, \lambda_{i}, r, \boldsymbol{\theta}, \mathbf{\Psi}, p, d\right)
$$

where the limit exists by the stationarity of the process. Similar to (15), it can be shown that for each $i=1, \ldots, m_{o}+1$,

$$
L\left(\lambda_{l}, \lambda_{u}, r, \boldsymbol{\theta}, \boldsymbol{\Psi}, p, d\right) \geq L\left(\lambda_{l}, \lambda_{u}, r_{0}^{(i)}, \boldsymbol{\theta}_{0}^{(i)}, \mathbf{\Psi}_{0}^{(i)}, p_{0}^{(i)}, d_{0}^{(i)}\right)
$$

for all $\left(\lambda_{l}, \lambda_{u}\right) \subseteq\left(\lambda_{0}^{(i-1)}, \lambda_{0}^{(i)}\right)$ and $r, \boldsymbol{\theta}, \boldsymbol{\Psi}, p$ and $d$, with equality holds only when $r \geq r_{0}^{(i)}, \boldsymbol{\theta}_{0}^{(i)} \subset \boldsymbol{\theta}$, $d=d_{0}$, and the $k$-th components of $\boldsymbol{\Psi}$ and $\boldsymbol{p}, \boldsymbol{\Psi}^{(k)}$ and $p^{(k)}$, respectively, satisfy $\left(\boldsymbol{\Psi}^{(k)}, p^{(k)}\right)=$ $\left(\left(\mathbf{\Psi}_{0}^{(i)}, \mathbf{0}_{s}\right), p_{0}^{(i)}+s\right)$ for some $s \geq 0$, provided that the $k$-th regime of $\boldsymbol{\theta}$ satisfies $\left(\theta^{(k-1)}, \theta^{(k)}\right) \subseteq$ $\left(\theta_{0}^{(i-1)}, \theta_{0}^{(i)}\right)$.

In Davis and Yau (2013), general conditions for consistency of change-points estimation in piecewise stationary time series models (including ARMA and GARCH models) are given. Therefore, to show Theorem 2, it suffices to verify the conditions in Theorem 5 of Davis and Yau (2013), i.e., their Assumption 1(1), 1*,2(2), 3 and 5. These assumptions are verified for ARMA models. In the following we show that TAR models does not satisfy all the assumptions, and we modify the proof to show the consistency.

Since the TAR model has a similar structure as the AR model, the statements about the likelihood function in each of the Assumption 1(1), 1*,2(2), 3 and 5 can be verified similarly. On the other hand, the statements about the derivatives of the likelihood function do not hold since the likelihood is not non-differentiable with respect to the parameters $r, d$ and p. Nevertheless, it can be seen that Theorem 1 and Corollary 1 in Davis and Yau (2013) hold for piecewise TAR models since the properties about the derivatives of the likelihood are not used in the proof. Thus 
the estimates of change-points satisfy

$$
\max _{i=1, \ldots, m_{0}} \min _{k=1, \ldots, \hat{m}_{n}}\left|\hat{\lambda}_{n}^{(k)}-\lambda_{0}^{(i)}\right| \stackrel{\text { a.s. }}{\rightarrow} 0
$$

and if, for fixed $i, i^{\prime}=\arg \min _{k=1, \ldots, \hat{m}_{n}}\left|\hat{\lambda}_{n}^{(k)}-\lambda_{0}^{(i)}\right|$, then

$$
\begin{array}{r}
\max _{j=1, \ldots, r_{0}^{(i)}} \min _{k=1, \ldots, \hat{r}_{n}^{\left(i^{\prime}\right)}}\left|\hat{\theta}_{n}^{\left(i^{\prime} k\right)}-\theta_{0}^{(i j)}\right| \stackrel{\text { a.s. }}{\rightarrow} 0, \\
\left\|\hat{\Psi}_{n}^{\left(i^{\prime} j_{i^{\prime}}^{\prime}\right)}-\left(\Psi_{0}^{(i j)}, \mathbf{0}_{\mathbf{s}}\right)\right\| \stackrel{\text { a.s. }}{\rightarrow} 0,
\end{array}
$$

where $j_{i^{\prime}}^{\prime}=\arg \min _{k=1, \ldots, \hat{r}_{n}^{\left(i^{\prime}\right)}}\left|\hat{\theta}_{n}^{\left(i^{\prime} k\right)}-\theta_{0}^{(i j)}\right|$ in (S.3), and $s$ is some non-negative integer. In other words, every estimated stationary segment is either approximately equal to or inside one true segment, and in each stationary segment, every estimated regime is either approximately equal to or inside one true regime. Moreover, in each regime, the order of the model will not be underestimated.

Next we establish the convergence rate of the estimators. We claim that for some $s \geq 0$,

$$
\begin{aligned}
\max _{i=1, \ldots, m_{0}} \min _{k=1, \ldots, \hat{m}_{n}}\left|\hat{\lambda}_{n}^{(k)}-\lambda_{0}^{(i)}\right| & =O_{p}\left(n^{-1}\right), \\
\max _{j=1, \ldots, r_{0}^{(i)}} \min _{k=1, \ldots, \hat{r}_{n}^{\left(i^{\prime}\right)}}\left|\hat{\theta}_{n}^{\left(i^{\prime} k\right)}-\theta_{0}^{(i j)}\right| & =O_{p}\left(n^{-1}\right), \\
\left\|\hat{\Psi}_{n}^{\left(i^{\prime} j_{i^{\prime}}^{\prime}\right)}-\left(\Psi_{0}^{(i j)}, \mathbf{0}_{s}\right)\right\| & =O_{p}\left(n^{-1 / 2}\right) .
\end{aligned}
$$

To show (S.5), similar to the proof in Proposition 5, it suffices to show that for each fixed $i$, there exists some $c_{1}$ such that

$$
P\left(\operatorname{MDL}\left(\hat{\boldsymbol{\lambda}}_{n}\right) \leq \operatorname{MDL}\left(\tilde{\boldsymbol{\lambda}}_{n}^{(i)}\right),\left|\lambda_{0}^{(i)}-\hat{\lambda}_{n}^{\left(i^{\prime}\right)}\right|>c_{1} n^{-1}\right) \rightarrow 0,
$$

where $\tilde{\boldsymbol{\lambda}}_{n}^{(i)}$ is the same as $\hat{\boldsymbol{\lambda}}_{n}$, except that $\hat{\lambda}_{n}^{\left(i^{\prime}\right)}$ is replaced by $\lambda_{0}^{(i)}$, and $\operatorname{MDL}\left(\hat{\boldsymbol{\lambda}}_{n}\right)$ and $\operatorname{MDL}\left(\tilde{\boldsymbol{\lambda}}_{n}^{(i)}\right)$ are the MDL evaluated at $\left(\hat{m}_{n}, \hat{\boldsymbol{\lambda}}_{n}, \hat{\boldsymbol{r}}_{n}, \hat{\boldsymbol{\theta}}_{n}, \hat{\boldsymbol{p}}_{n}, \hat{\mathbf{\Psi}}_{n}, \hat{\mathbf{d}}_{n}\right)$ and $\left(\hat{m}_{n}, \tilde{\boldsymbol{\lambda}}_{\boldsymbol{n}}^{(i)}, \hat{\boldsymbol{r}}_{n}, \hat{\boldsymbol{\theta}}_{n}, \hat{\boldsymbol{p}}_{n}, \hat{\mathbf{\Psi}}_{n}, \hat{\mathbf{d}}_{n}\right)$, respectively. To show (S.8), note that $2\left(\operatorname{MDL}\left(\hat{\boldsymbol{\lambda}}_{n}\right)-\operatorname{MDL}\left(\tilde{\boldsymbol{\lambda}}_{n}^{(i)}\right)\right)$ is equal to either one of

$$
\begin{aligned}
& i) L_{n}\left(\lambda_{0}^{(i)}, \hat{\lambda}_{n}^{\left(i^{\prime}\right)}, \hat{r}_{n}^{\left(i^{\prime}\right)}, \hat{\boldsymbol{\theta}}_{n}^{\left(i^{\prime}\right)}, \hat{\mathbf{\Psi}}_{n}^{\left(i^{\prime}\right)}, \hat{p}_{n}^{\left(i^{\prime}\right)}, \hat{d}_{n}^{\left(i^{\prime}\right)}\right)-L_{n}\left(\lambda_{0}^{(i)}, \hat{\lambda}_{n}^{\left(i^{\prime}\right)}, \hat{r}_{n}^{\left(i^{\prime}+1\right)}, \hat{\boldsymbol{\theta}}_{n}^{\left(i^{\prime}+1\right)}, \hat{\mathbf{\Psi}}_{n}^{\left(i^{\prime}+1\right)}, \hat{p}_{n}^{\left(i^{\prime}+1\right)}, \hat{d}_{n}^{\left(i^{\prime}+1\right)}\right), \\
& i i) L_{n}\left(\hat{\lambda}_{n}^{\left(i^{\prime}\right)}, \lambda_{0}^{(i)}, \hat{r}_{n}^{\left(i^{\prime}+1\right)}, \hat{\boldsymbol{\theta}}_{n}^{\left(i^{\prime}+1\right)}, \hat{\mathbf{\Psi}}_{n}^{\left(i^{\prime}+1\right)}, \hat{p}_{n}^{\left(i^{\prime}+1\right)}, \hat{d}_{n}^{\left(i^{\prime}+1\right)}\right)-L_{n}\left(\hat{\lambda}_{n}^{\left(i^{\prime}\right)}, \lambda_{0}^{(i)}, \hat{r}_{n}^{\left(i^{\prime}\right)}, \hat{\boldsymbol{\theta}}_{n}^{\left(i^{\prime}\right)}, \hat{\mathbf{\Psi}}_{n}^{\left(i^{\prime}\right)}, \hat{p}_{n}^{\left(i^{\prime}\right)}, \hat{d}_{n}^{\left(i^{\prime}\right)}\right) .
\end{aligned}
$$

From (S.3), (S.4) and (S.2), it can be shown that both terms in i) and ii) are strictly positive with probability approaching 1 when $\left|\lambda_{0}^{(i)}-\hat{\lambda}_{n}^{\left(i^{\prime}\right)}\right|>c_{1} n^{-1}$ and $c_{1}$ sufficiently large, yielding (S.8). 
Next we show (S.6). It suffices to establish that, for $i=1, \ldots, m_{0}, j=1, \ldots, r_{0}^{(i)}$,

$$
P\left(\operatorname{MDL}\left(\hat{\boldsymbol{\theta}}_{n}\right) \leq \operatorname{MDL}\left(\tilde{\boldsymbol{\theta}}_{n}^{(i, j)}\right),\left|\lambda_{0}^{(i)}-\hat{\lambda}_{n}^{\left(i^{\prime}\right)}\right|<c_{1} n^{-1},\left|\theta_{0}^{(i, j)}-\hat{\theta}_{n}^{\left(i^{\prime}, j_{i^{\prime}}^{\prime}\right)}\right|>c_{2} n^{-1}\right) \rightarrow 0,
$$

for some $c_{2}>0$, where $\tilde{\boldsymbol{\theta}}_{n}^{(i, j)}$ is the same as $\hat{\boldsymbol{\theta}}_{n}$, except that $\hat{\theta}_{n}^{\left(i^{\prime}, j_{i^{\prime}}^{\prime}\right)}$ is replaced by $\theta_{0}^{(i, j)}, \operatorname{MDL}\left(\hat{\boldsymbol{\theta}}_{n}\right)$ and $\operatorname{MDL}\left(\tilde{\boldsymbol{\theta}}_{n}^{(i, j)}\right)$ are the MDL evaluated at $\left(\hat{m}_{n}, \hat{\boldsymbol{\lambda}}_{n}, \hat{\boldsymbol{r}}_{n}, \hat{\boldsymbol{\theta}}_{n}, \hat{\boldsymbol{p}}_{n}, \hat{\mathbf{\Psi}}_{n}, \hat{\mathbf{d}}_{n}\right)$ and $\left(\hat{m}_{n}, \hat{\boldsymbol{\lambda}}_{n}, \hat{\boldsymbol{r}}_{n}, \tilde{\boldsymbol{\theta}}_{n}^{(i, j)}, \hat{\boldsymbol{p}}_{n}, \hat{\mathbf{\Psi}}_{n}, \hat{\mathbf{d}}_{n}\right)$, respectively. Using similar arguments in (S.8) and (S.14), (S.9) follows. Lastly, with the fast convergence rate of $\hat{\boldsymbol{\lambda}}_{n}$ and $\hat{\boldsymbol{\theta}}_{n}$, (S.7) can be shown by standard argument for maximum likelihood estimators.

Since the number of change-points, the number of thresholds, and the order of the model will not be underestimated, to show the consistency, it remains to show that these parameters cannot be overestimated. Let $\tilde{\boldsymbol{\lambda}}_{n}=\left(\tilde{\lambda}_{n}^{(1)}, \ldots, \tilde{\lambda}_{n}^{\left(m_{o}\right)}\right)$ and $\tilde{\boldsymbol{\theta}}_{n}=\left(\tilde{\boldsymbol{\theta}}_{n}^{(1)}, \ldots, \tilde{\boldsymbol{\theta}}_{n}^{\left(m_{o}+1\right)}\right)$, with $\tilde{\boldsymbol{\theta}}_{n}^{(i)}=$ $\left(\tilde{\theta}_{n}^{(i 1)}, \ldots, \tilde{\theta}_{n}^{\left(i r_{0}^{(i)}\right)}\right)$, be sequences satisfying $\left|\tilde{\lambda}_{n}^{(i)}-\lambda_{0}^{(i)}\right|=O\left(n^{-1}\right)$ and $\left|\tilde{\theta}_{n}^{(i j)}-\theta_{0}^{(i j)}\right|=O\left(n^{-1}\right)$. For any integers $s_{1}=1, \ldots, M-m_{o}$ and $s_{2}^{(i)}=1 \ldots, R-r_{0}^{(i)}, i=1, \ldots, m_{0}+1$, where $M$ and $R$ are the assumed upper bounds of the change-points and thresholds, respectively, define $\boldsymbol{r}_{0}=$ $\left(r_{0}^{(1)}, \ldots, r_{0}^{\left(m_{o}+1\right)}\right)$ and $\boldsymbol{r}_{s}=\left(r_{0}^{(1)}+s_{2}^{(1)}, \ldots, r_{0}^{\left(m_{o}+1\right)}+s_{2}^{\left(m_{o}+1\right)}\right)$. Similar to $(22)$, it suffices to consider the quantity

$$
\min _{\boldsymbol{\lambda} \supset \tilde{\boldsymbol{\lambda}}_{n}, \boldsymbol{\theta} \supset \tilde{\boldsymbol{\theta}}_{\boldsymbol{n}}}\left[\frac{2}{n} \operatorname{MDL}\left(m_{o}+s_{1}, \boldsymbol{\lambda}, \boldsymbol{r}_{s}, \boldsymbol{\theta}, \mathbf{p}\right)\right]-\frac{2}{n} \mathrm{MDL}_{0}\left(m_{0}, \tilde{\boldsymbol{\lambda}}_{\boldsymbol{n}}, \boldsymbol{r}_{0}, \tilde{\boldsymbol{\theta}}_{\boldsymbol{n}}, \mathbf{p}_{0}\right)
$$

where $\mathrm{MDL}_{0}$ is the MDL with $\mathrm{AR}$ coefficients evaluated at the true value and $\mathbf{p}$ is the vector of $\mathrm{AR}$ orders that do not underestimate the true orders. Using (S.7), similar expansions as in (23) and (24) can be applied to show that the quantity in (S.10) is positive with probability approaching 1 if $s_{1}>0$ or $s_{2}^{(i)}>0$, or any model order is overestimated. This concludes the consistency of MDL procedure on TAR models with structural breaks .

\section{S.3 Proofs of Propositions}

Proof of Proposition 1. The proofs of Proposition 1 follows similar arguments in Propositions 1, 2 and 4 of Davis and Yau (2013). We outline the proof for $k=0$; the case $k=1,2$ follow similarly. 
First, by the compactness of the parameter space $\bar{\Psi}_{j}$ and the ergodic theorem, we have

$$
\sup _{\Psi_{j} \in \bar{\Psi}_{j}}\left|\frac{1}{n} L_{n}\left(\Psi_{j}, \theta_{l}, \theta_{u}, p_{j}, d\right)-L\left(\Psi_{j}, \theta_{l}, \theta_{u}, p_{j}, d\right)\right| \stackrel{a . s .}{\longrightarrow} 0
$$

for fixed $\theta_{l}$ and $\theta_{u}$ in $\left[\theta_{o}^{(j-1)}, \theta_{o}^{(j)}\right]$. In particular, (S.11) holds with $\theta_{l}=r_{1}$ and $\theta_{u}=r_{2}$ for any rational numbers $r_{1}, r_{2}$ in $\left[\theta_{o}^{(j-1)}, \theta_{o}^{(j)}\right]$. Let $B_{r_{1}, r_{2}}$ be the probability one set such that (S.11) holds. Note that $P\left(\cap_{r_{1}, r_{2}} B_{r_{1}, r_{2}}\right)=1$ where the intersection is over all pairs of distinct rational numbers $r_{1}, r_{2}$ in $\left[\theta_{o}^{(j-1)}, \theta_{o}^{(j)}\right]$. Thus equation (S.11) holds uniformly on all subintervals nested inside $\left[\theta_{o}^{(j-1)}, \theta_{o}^{(j)}\right]$ with rational endpoints. Next, note that for any $\epsilon>0$ and $\theta \in\left[\theta_{o}^{(j-1)}, \theta_{o}^{(j)}\right]$, there exists a rational $r<\theta$ such that

$$
\begin{aligned}
& \sup _{\Psi_{j} \in \bar{\Psi}_{j}}\left|\frac{1}{n} L_{n}\left(\Psi_{j}, \theta_{o}^{(j-1)}, \theta, p_{j}, d\right)-L\left(\Psi_{j}, \theta_{o}^{(j-1)}, r, p_{j}, d\right)\right| \\
\leq & \sup _{\Psi_{j} \in \bar{\Psi}_{j}}\left|\frac{1}{n} \sum_{i=1}^{n}\left(\log \left(2 \pi \sigma^{2}\right)+\frac{\left(X_{t}-\Phi_{j}^{\prime} \mathbf{X}_{t-1}\right)^{2}}{\sigma^{2}}\right) I\left(r<X_{t-d} \leq \theta\right)\right| \\
\leq & \sup _{\Psi_{j} \in \bar{\Psi}_{j}}\left|\left(\log \left(2 \pi \sigma^{2}\right)+E\left(\frac{\left(X_{t}-\Phi_{j}^{\prime} \mathbf{X}_{t-1}\right)^{2}}{\sigma^{2}}\right)\right) Q(r, \theta)+\frac{\epsilon}{2}\right|<\epsilon,
\end{aligned}
$$

for $n$ sufficiently large. It follows that, for the left quantity in (S.11), $\left(\theta_{l}, \theta_{u}\right)$ can be arbitrarily closely approximated by some rational pair $\left(r_{1}, r_{2}\right)$, which in turn implies that (S.11) holds uniformly in $\left(\theta_{l}, \theta_{u}\right) \subseteq\left[\theta_{o}^{(j-1)}, \theta_{o}^{(j)}\right]$. Similar argument shows that if $\theta_{l}$ and $\theta_{u}$ are outside $\left[\theta_{o}^{(j-1)}, \theta_{o}^{(j)}\right]$ within a shrinking neighborhood, the effect on the likelihood function is negligible. Thus the uniform convergence of Proposition 1 follows.

Proof of Proposition 2. By the definition of $\hat{\mathbf{\Psi}}_{n} \equiv \hat{\mathbf{\Psi}}_{n}\left(\theta_{l}, \theta_{u}, p, d\right)$, we have

$$
L_{n}\left(\hat{\mathbf{\Psi}}_{n}, \theta_{l}, \theta_{u}, p, d\right) \leq L_{n}\left(\mathbf{\Psi}_{*}, \theta_{l}, \theta_{u}, p, d\right)
$$

for every $\theta_{l}, \theta_{u}, p, d$ and $n$. On the other hand, by the definition of $\boldsymbol{\Psi}_{*} \equiv \boldsymbol{\Psi}_{*}\left(\theta_{l}, \theta_{u}, p, d\right)$ and 
Proposition 1, we have

$$
\begin{aligned}
0 \leq & L\left(\hat{\mathbf{\Psi}}_{n}, \theta_{l}, \theta_{u}, p, d\right)-L\left(\mathbf{\Psi}_{*}, \theta_{l}, \theta_{u}, p, d\right) \\
\leq & \left|L\left(\hat{\mathbf{\Psi}}_{n}, \theta_{l}, \theta_{u}, p, d\right)-\frac{1}{n} L_{n}\left(\hat{\mathbf{\Psi}}_{n}, \theta_{l}, \theta_{u}, p, d\right)\right|+\left|\frac{1}{n} L\left(\hat{\mathbf{\Psi}}_{n}, \theta_{l}, \theta_{u}, p, d\right)-\frac{1}{n} L_{n}\left(\mathbf{\Psi}_{*}, \theta_{l}, \theta_{u}, p, d\right)\right| \\
& +\left|\frac{1}{n} L_{n}\left(\mathbf{\Psi}_{*}, \theta_{l}, \theta_{u}, p, d\right)-L\left(\mathbf{\Psi}_{*}, \theta_{l}, \theta_{u}, p, d\right)\right| \\
& \stackrel{\text { a.s. }}{\rightarrow} 0,
\end{aligned}
$$

uniformly on $\theta_{l}, \theta_{u}$. By the convexity of $L, \Psi_{*}$ is the unique minimum of $L(\cdot)$, hence Proposition 2 follows.

Proof of Proposition 3. a) Let $B$ be the probability one set in which Propositions 1 and 2 hold. For each $\omega \in B$, suppose on the contrary that the number of thresholds is underestimated or (16) does not hold. Then, by the compactness of the parameter space and Proposition 2, there exists a subsequence $\left\{n_{k}\right\}$ such that $\hat{r}_{n_{k}} \rightarrow r_{*}, \hat{d}_{n_{k}} \rightarrow d_{*}, \hat{\boldsymbol{\theta}}_{n_{k}} \rightarrow \boldsymbol{\theta}_{*} \neq \boldsymbol{\theta}_{0}$ with $\theta_{0}^{(i-1)} \leq \theta_{*}^{(j-1)}<$ $\theta_{0}^{(i)}<\cdots<\theta_{0}^{(i+k)}<\theta_{*}^{(j)} \leq \theta_{0}^{(i+k+1)}$ for some integers $i, j$ and $k$, and $\hat{\mathbf{p}}_{n_{k}} \rightarrow \mathbf{p}_{*}$, for some $r_{*}, \boldsymbol{\theta}_{*}$ and $\mathbf{p}_{*}$. In this case, for sufficiently large $n_{k}$, the estimated $j$-th regime contains observations from at least two different regimes. For notational simplicity we write $n$ instead of $n_{k}$. Partition $L_{n}$ with respect to the true configuration of the regimes, we have

$$
\begin{aligned}
L_{n}\left(\hat{\Psi}_{n}^{(j)}, \hat{\theta}_{n}^{(j)}, \hat{\theta}_{n}^{(j+1)}, \hat{p}_{n}^{(j)}, \hat{d}_{n}\right)= & L_{n}\left(\hat{\Psi}_{n}^{(j)}, \hat{\theta}_{n}^{(j)}, \theta_{0}^{(i)}, \hat{p}_{n}^{(j)}, \hat{d}_{n}\right)+\sum_{l=i}^{i+k-1} L_{n}\left(\hat{\Psi}_{n}^{(j)}, \theta_{0}^{(l)}, \theta_{0}^{(l+1)}, \hat{p}_{n}^{(j)}, \hat{d}_{n}\right) \\
& +L_{n}\left(\hat{\Psi}_{n}^{(j)}, \theta_{0}^{(i+k)}, \hat{\theta}_{n}^{(j+1)}, \hat{p}_{n}^{(j)}, \hat{d}_{n}\right) .
\end{aligned}
$$

Note from Proposition 2 that, in the $j$-th regime, we have $\hat{\Psi}_{n}^{(j)} \rightarrow \Psi_{*}^{(j)}$ for some $\Psi_{*}^{(j)}$. From (15), we have that $L\left(\Psi_{0}^{(j)}, \theta_{l}, \theta_{u}, p_{0}^{(j)}, d_{0}\right) \leq L\left(\Psi_{*}^{(j)}, \theta_{l}, \theta_{u}, p_{*}, d_{*}\right)$ for all $j=i, \ldots, i+k+1$ and $\left(\theta_{l}, \theta_{u}\right) \subseteq\left(\theta_{0}^{(j-1)}, \theta_{0}^{(j)}\right)$. Therefore

$$
\begin{aligned}
\lim _{n \rightarrow \infty} \frac{1}{n} L_{n}\left(\hat{\Psi}_{n}^{(j)}, \hat{\theta}_{j}, \theta_{0}^{(i)}, \hat{p}_{n}^{(j)}, \hat{d}_{n}\right) & \geq L\left(\Psi_{0}^{(i)}, \theta_{*}^{(j-1)}, \theta_{0}^{(i)}, p_{0}^{(j)}, d_{0}\right), \\
\lim _{n \rightarrow \infty} \frac{1}{n} L_{n}\left(\hat{\Psi}_{n}^{(j)}, \theta_{0}^{(l)}, \theta_{0}^{(l+1)}, \hat{p}_{n}^{(j)}, \hat{d}_{n}\right) & \geq L\left(\Psi_{0}^{(l+1)}, \theta_{0}^{(l)}, \theta_{0}^{(l+1)}, p_{0}^{(l+1)}, d_{0}\right), \\
\lim _{n \rightarrow \infty} \frac{1}{n} L_{n}\left(\hat{\Psi}_{n}^{(j)}, \theta_{0}^{(i+k)}, \hat{\theta}_{j+1}, \hat{p}_{n}^{(j)}, \hat{d}_{n}\right) & \geq L\left(\Psi_{0}^{(i+k+1)} \theta_{0}^{(i+k)}, \theta_{*}^{(j)}, p_{0}^{(i+k+1)}, d_{0}\right) .
\end{aligned}
$$


Note that strict inequalities hold for at least one of the above equations since $\Psi_{j}^{*}$ cannot correctly specify the model for all different regimes. Thus, we have

$$
\begin{array}{r}
\lim _{n \rightarrow \infty} \frac{1}{n} L_{n}\left(\hat{\Psi}_{n}^{(j)}, \hat{\theta}_{n}^{(j)}, \hat{\theta}_{n}^{(j+1)}, \hat{p}_{n}^{(j)}, \hat{d}_{n}\right)>L\left(\Psi_{0}^{(i)}, \theta_{*}^{(j-1)}, \theta_{0}^{(i)}, p_{0}^{(j)}, d_{0}\right) \\
+\sum_{l=i}^{i+k-1} L\left(\Psi_{0}^{(l+1)}, \theta_{0}^{(l)}, \theta_{0}^{(l+1)}, p_{0}^{(l+1)}, d_{0}\right)+L\left(\Psi_{0}^{(i+k+1)} \theta_{0}^{(i+k)}, \theta_{*}^{(j)}, p_{0}^{(i+k+1)}, d_{0}\right) .
\end{array}
$$

Consequently, for sufficiently large $n$,

$$
\begin{aligned}
& \frac{2}{n} \operatorname{MDL}\left(\hat{r}_{n}, \hat{\theta}_{\mathbf{n}}, \hat{\mathbf{p}}_{\mathbf{n}}\right) \\
> & \left.\frac{2}{n} \operatorname{Pen}\left(\hat{r}_{n}, \hat{\mathbf{p}}_{\mathbf{n}}\right)+\sum_{l=1}^{\hat{r}_{n}+1} L\left(\Psi_{0}^{(l+1)}, \theta_{0}^{(l)}, \theta_{0}^{(l+1)}, p_{0}^{(l+1)}, d_{0}\right) \quad \text { [Using }(\mathrm{S} .12)\right] \\
= & \frac{2}{n} \operatorname{MDL}\left(r_{*}, \theta_{\mathbf{0}}, \mathbf{p}_{\mathbf{0}}\right)+o_{p}(1) .
\end{aligned}
$$

For $n$ sufficiently large, the last quantity is greater than $\frac{2}{n} \operatorname{MDL}\left(\hat{r}, \hat{\theta}_{\mathbf{n}}, \hat{\mathbf{p}}_{\mathbf{n}}\right)$ by the definition of the estimator. Thus contradiction arises and the proof of a) is completed.

On the other hand, suppose that (16) holds but $d_{*} \neq d_{0}$ or the estimated model underestimates the true model in some of the segments. In the latter case, there exists some $j>0$ such that $\theta_{0}^{(j-1)} \leq \theta_{*}^{(j-1)}<\theta_{*}^{(j)} \leq \theta_{0}^{(j)}$ and $\hat{p}_{n}^{(j)}<p_{0}^{(j)}$ hold. In both situations, it follows that along some subsequence,

$$
\begin{aligned}
\frac{1}{n} L_{n}\left(\hat{\Psi}_{n}, \hat{\theta}_{n}^{(j-1)}, \hat{\theta}_{n}^{(j)}, \hat{p}_{n}^{(j)}, \hat{d}_{n}\right) & \rightarrow L\left(\Psi_{*}^{(j+1)}, \theta_{0}^{(j)}, \theta_{0}^{(j+1)}, p_{*}^{(j+1)}, d_{*}\right) \\
& >L\left(\Psi_{0}^{(j+1)}, \theta_{0}^{(j)}, \theta_{0}^{(j+1)}, p_{0}^{(j+1)}, d_{0}\right)
\end{aligned}
$$

and contradiction arises. Thus the estimated model does not underestimate the true model. This completes the proof of Proposition 3.

Proof of Proposition 4. First, (17) follows from the same arguments in establishing equation (4.4) in Chan (1993). Next, using the definition of $l_{t}$ and Proposition 3, the quantity inside the supremum in (18) can be expressed as

$$
\begin{aligned}
& \left\{\sum _ { t = d _ { 0 } + 1 } ^ { n } \left[\log \sigma_{j+1}^{2}-\log \sigma_{j}^{2}+\epsilon_{t}^{2}\left(\frac{1}{\sigma_{j+1}^{2}}-\frac{1}{\sigma_{j}^{2}}\right)-\frac{1}{\sigma_{j}^{2}}\left(\Psi_{0}^{(j+1)}-\Psi_{0}^{(j)}\right)^{\prime} \mathbf{X}_{t-1} \mathbf{X}_{t-1}^{\prime}\left(\Psi_{0}^{(j+1)}-\Psi_{0}^{(j)}\right)\right.\right. \\
& \left.\left.-\frac{2}{\sigma_{j}^{2}} \epsilon_{t}\left(\Psi_{0}^{(j+1)}-\Psi_{0}^{(j)}\right)^{\prime} \mathbf{X}_{t-1}\right] I\left(\theta_{0}^{(j)}-z<X_{t-d_{0}} \leq \theta_{0}^{(j)}\right)\right\}\left(1+o_{p}(1)\right) .
\end{aligned}
$$


Combining (17), (S.13) and the facts that $\left(\Psi_{0}^{(j+1)}-\Psi_{0}^{(j)}\right)^{\prime} \mathbf{X}_{t-1} \mathbf{X}_{t-1}^{\prime}\left(\Psi_{0}^{(j+1)}-\Psi_{0}^{(j)}\right) \geq 0$ and $\log \sigma_{j+1}^{2}-$ $\log \sigma_{j}^{2}+\sigma_{j+1}^{2}\left(\frac{1}{\sigma_{j+1}^{2}}-\frac{1}{\sigma_{j}^{2}}\right) \leq 0$ (follows from the inequality $x-1 \geq \log x$ ), it can be seen that the quantity in (S.13) is non-positive. Since $\left(\Psi_{0}^{(j)}, \sigma_{j}^{2}\right) \neq\left(\Psi_{0}^{(j+1)}, \sigma_{j+1}^{2}\right)$, strict inequality hold and thus (18) follows. Finally, (19) can be shown similarly.

Proof of Proposition 5. From Proposition 3 we know that $\hat{r}_{n} \geq r_{0}$ and for each $\theta_{0}^{(i)}$ there exists a $\hat{\theta}_{n}^{\left(i^{\prime}\right)}$ such that $\left|\hat{\theta}_{n}^{\left(i^{\prime}\right)}-\theta_{0}^{(i)}\right|=o(1)$, a.s., where $1 \leq i^{\prime} \leq \hat{r}_{n}$. To prove (20), it suffices to show that for each $\epsilon>0$ and $i=1, \ldots, m_{0}$, there exists a $c$ such that

$$
P\left(\exists i,\left|\theta_{0}^{(i)}-\hat{\theta}_{n}^{\left(i^{\prime}\right)}\right|>c n^{-1}\right)<\epsilon .
$$

By definition, the parameter $\left(\hat{r}_{n}, \hat{d}_{n}, \hat{\boldsymbol{\theta}}_{n}, \hat{\boldsymbol{p}}_{n}, \hat{\mathbf{\Psi}}_{n}\right)$ minimizes the MDL. Thus, we have for each $j=1, \ldots, r_{0}$ that $\operatorname{MDL}\left(\hat{\boldsymbol{\theta}}_{n}\right) \leq \operatorname{MDL}\left(\tilde{\boldsymbol{\theta}}_{n}^{(i)}\right)$, where $\tilde{\boldsymbol{\theta}}_{n}^{(i)}$ is the same as $\hat{\boldsymbol{\theta}}_{n}$, except that $\hat{\theta}_{n}^{\left(i^{\prime}\right)}$ is replaced by $\theta_{0}^{(i)}$, and $\operatorname{MDL}\left(\hat{\boldsymbol{\theta}}_{n}\right)$ and $\operatorname{MDL}\left(\tilde{\boldsymbol{\theta}}_{n}^{(i)}\right)$ are the $\operatorname{MDL}$ evaluated at $\left(\hat{r}_{n}, \hat{d}_{n}, \hat{\boldsymbol{\theta}}_{n}, \hat{\boldsymbol{p}}_{n}, \hat{\mathbf{\Psi}}_{n}\right)$ and $\left(\hat{r}_{n}, \hat{d}_{n}, \tilde{\boldsymbol{\theta}}_{n}^{(i)}, \hat{\boldsymbol{p}}_{n}, \hat{\mathbf{\Psi}}_{n}\right)$, respectively. Therefore, it in turns suffices to show that for each $i=1, \ldots, r_{0}$,

$$
P\left(\operatorname{MDL}\left(\hat{\boldsymbol{\theta}}_{n}\right) \leq \operatorname{MDL}\left(\tilde{\boldsymbol{\theta}}_{n}^{(i)}\right),\left|\theta_{0}^{(i)}-\hat{\theta}_{n}^{\left(i^{\prime}\right)}\right|>c n^{-1}\right)<\epsilon .
$$

Given that $\left|\theta_{0}^{(i)}-\hat{\theta}_{n}^{\left(i^{\prime}\right)}\right|>c n^{-1}$, the quantity $\operatorname{MDL}\left(\tilde{\boldsymbol{\theta}}_{n}^{(i)}\right)-\operatorname{MDL}\left(\hat{\boldsymbol{\theta}}_{n}\right)$ is equal to either one of

$$
\begin{array}{ll}
\text { i) } \quad \sum_{t=\hat{d}_{n}+1}^{n}\left(l_{t}\left(\hat{\Psi}_{n}^{(i+1)}\right)-l_{t}\left(\hat{\Psi}_{n}^{(i)}\right)\right) I\left(\theta_{0}^{(i)}<X_{t-\hat{d}_{n}} \leq \hat{\theta}_{n}^{(i)}\right), \\
\text { ii) } \quad \sum_{t=\hat{d}_{n}+1}^{n}\left(l_{t}\left(\hat{\Psi}_{n}^{(i)}\right)-l_{t}\left(\hat{\Psi}_{n}^{(i+1)}\right)\right) I\left(\hat{\theta}_{n}^{(i)}<X_{t-\hat{d}_{n}} \leq \theta_{0}^{(i)}\right) .
\end{array}
$$

By Propositions 2 and 4, for sufficiently large $c$, both terms in (S.15)i)ii) are negative with probability approaching 1 , that is, $\operatorname{MDL}\left(\hat{\boldsymbol{\theta}}_{n}\right)>\operatorname{MDL}\left(\tilde{\boldsymbol{\theta}}_{n}^{(i)}\right)$ with probability approaching 1, yielding (S.14).

Given the fast convergence rate of $\hat{\theta}_{n}^{(j)}$ s, the asymptotic properties of $\hat{\Psi}_{n}^{(j)}$ can be derived as if $\theta_{0}^{(j)} \mathrm{s}$ are known. Thus the $n^{-1 / 2}$ convergence rate of $\hat{\Psi}_{n}^{(j)}$ follows from standard argument of maximum likelihood estimation of AR models, completing the proof of Proposition 5. 


\section{References}

Chan, K. S. (1993) Consistency and limiting distribution of the least squares estimator of a threshold autoregressive model. The Annals of Statistics, 21, 520-533.

Davis, R. A. and Yau, C. Y. (2013) Consistency of minimum description length model selection for piecewise stationary time series models. Electronic Journal of Statistics, 7, 381-411. 